\title{
Escher in color space: Individual-differences multidimensional scaling of color dissimilarities collected with a gestalt formation task
}

\author{
DAVID BIMLER and JOHN KIRKLAND \\ Massey University, Palmerston North, New Zealand \\ and \\ SHAUN PICHLER \\ Loyola University, Chicago, Illinois
}

\begin{abstract}
The structure of color perception can be examined by collecting judgments about color dissimilarities. In the procedure used here, stimuli are presented three at a time on a computer monitor and the spontaneous grouping of most-similar stimuli into gestalts provides the dissimilarity comparisons. Analysis with multidimensional scaling allows such judgments to be pooled from a number of observers without obscuring the variations among them. The anomalous perceptions of color-deficient observers produce comparisons that are represented well by a geometric model of compressed individual color spaces, with different forms of deficiency distinguished by different directions of compression. The geometrical model is also capable of accommodating the normal spectrum of variation, so that there is greater variation in compression parameters between tests on normal subjects than in those between repeated tests on individual subjects. The method is sufficiently sensitive and the variations sufficiently large that they are not obscured by the use of a range of monitors, even under somewhat loosely controlled conditions.
\end{abstract}

Ideally, a system for specifying colors such as Munsell notation or OSA space would have independent axes (free from interactions), and equal color dissimilarities would be represented by equal steps along these axes. In numerous studies - too many to list here-researchers have set out to gauge the adequacy of such systems by applying multidimensional scaling (MDS) to information about the perceived dissimilarities among color stimuli in order to locate them in a spatial model or map (see Indow, 1988, for a review; see also Taylor \& Billmeyer, 1988). The adequacy of 3-D Euclidean space has been tested with the same approach (Wuerger, Maloney, \& Krauskopf, 1995). The perceived dissimilarities are not entirely consistent among observers (Wish \& Carroll, 1974), but these variations have been of secondary concern. In particular, color-visiondeficient observers are excluded from the majority of studies. That of Helm (1964) is an exception.

Helm (1964) employed the method of triads, which has a long history within this research tradition as a way of

We are indebted to the editor and to the anonymous reviewers of earlier versions of this paper for their constructive comments. Correspondence concerning this article should be addressed to J. Kirkland, Department of Health and Human Development, Massey University, Private Bag 11-222, Palmerston North, New Zealand 0644-6-3586099(e-mail: j.kirkland@massey.ac.nz). eliciting the dissimilarity data. The task of quantifying interstimulus dissimilarity, which is often difficult when the stimuli are far removed from each other, is replaced with an easier comparison task: Which dissimilarity is the smallest? Pioneered by Richardson (1938) and Torgerson (1958), the triadic method yields stable results across retests of a single subject (Wright, 1965). However, the cognitive processing involved in dissimilarity comparisons complicates the picture: Subjects may access their knowledge of color semantics to respond to the triad, having coded the stimuli as color names (Roberson \& Davidoff, 2000). Stalmeier and de Weert $(1988,1994)$ combined computerized display of the stimuli with an ingenious forcedchoice technique, in which Star of David gestalt formation replaces conscious decisions. ${ }^{1}$ This reduces both cognitive involvement and the effort required by the task.

Here, we extend the technique, enhancing the effect of gestalt formation by repeating the basic elements. The computer screen is covered with an Escher-like tessellation (Figure 1) of lozenges in three different alignments, colored according to the stimuli constituting each triad. Grouping of similarly colored lozenges causes a pattern of stripes to emerge spontaneously from the tessellation, with an alignment or overall sense of direction that depends on which two stimuli are most similar (there are three possible pairs and three possible alignments). Although the technique works with a large number of stimuli, permitting the con- 


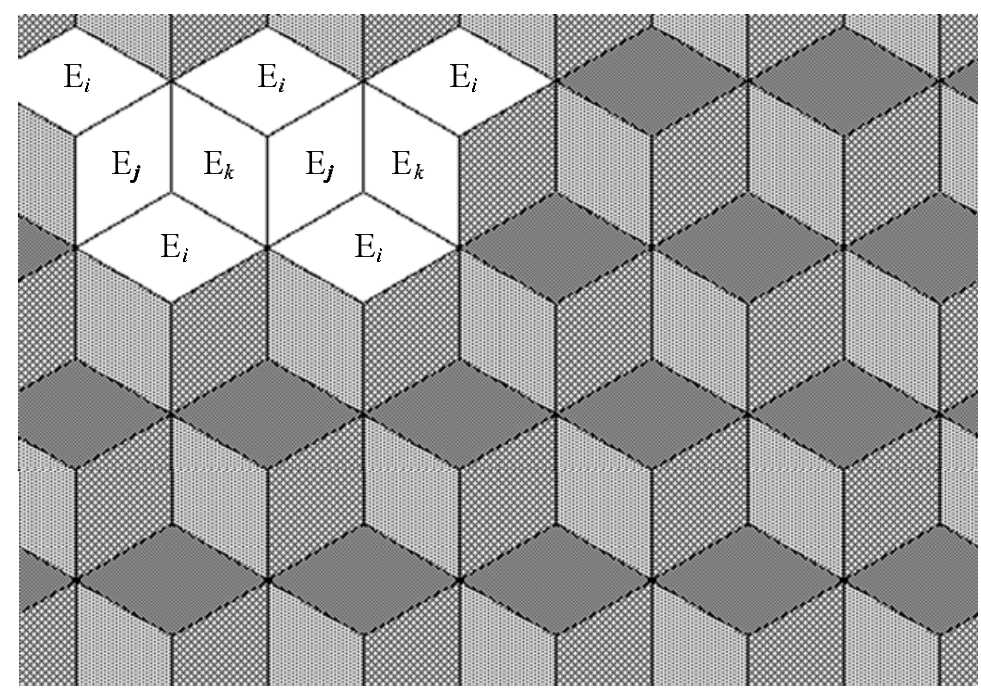

Figure 1. Tessellation used for collecting triadic dissimilarities. According to their orientation, lozenges are colored with one of three color stimuli $\left(\mathbf{E}_{i}, \mathbf{E}_{j}\right.$, and $\mathbf{E}_{k}$ ), which constitute a triad.

struction of a fine-grained map of color space, our own research has focused on individual variations.

It is known that, in a first approximation, the color perception of red-green-deficient (RGD) observers can be accommodated in a geometrical framework, in which individual color spaces are deformed versions of a normal consensus space (see, e.g., Farnsworth, 1943). A family of allowable deformations is defined and an observer's data are matched by adjusting the appropriate parameters . A first test of the technique described here is whether it replicates earlier applications of this geometrical framework in which different forms of data were analyzed (Bimler, Kirkland, \& Jacobs, 2000; Paramei, Bimler, \& Cavonius, 2001).

Departures from the consensus color space also appear among normal trichromats (NTs). Wish and Carroll (1974) reanalyzed earlier data (Helm, 1964; Indow \& Kanazawa, 1960) and found that NT subjects were ranged across a spectrum by their parameters (i.e., dimensional saliences). A similar range emerged from the two data sets collected by Stalmeier and de Weert $(1988,1994)$, whose subjects provided conventional triadic comparisons as well as gestalt fusion responses.

We have sought to establish whether such variations are genuine or are artifacts of the analysis arising from random fluctuations in the data. If they are genuine subject variations, the parameters describing subject performance should vary less when subjects are retested than when subjects are compared with each other. Assuming that individual variations are robust, also at issue is their magnitude. It is easier to justify investigating them if they emerge above the noise in conditions that are not scrupulously controlled.

The data we present below were collected from a range of computers under conditions that were controlled poorly, if at all. In some cases, the tests were self-administered by subjects who received the triad-presentation software by e-mail, ran it on their PCs, and delivered their data by email. The questions are whether RGDs can be classified accurately under these unpromising circumstances and, more generally, whether observer parameters can be replicated. Assuming that the present range of conditions and hardware is representative of variations to be generally encountered, a positive answer can be extrapolated to a range of contexts in which variants of the gestalt technique may be useful tools. Software can be written (e.g., in Java) to present color triads on a Web page: Although administering the procedure on the Web does not yet allow sufficient control for definitive purposes, it may suffice for screening.

The effect of relaxing the conditions is to enlarge the available subject population, allowing research that would otherwise be impractical. This is not to assert that the present methodology is unaffected by broad variations in conditions such as monitor characteristics. We did not quantify the variations (which may in practice have been small); it is enough that they were representative.

\section{METHOD}

\section{Procedure}

The stimuli were displayed three at a time using the tessellation shown in Figure 1. The length of each lozenge edge was 25 pixels, with the monitor resolution set to $640 \times 480$ pixels (approximately $1.5^{\circ}$ on a 17 -in. screen at a comfortable viewing distance of $50 \mathrm{~cm}$ ). The lozenges were separated by a fine black line ( 1 pixel wide) to remove any influence on gestalt formation from the distinctness of borders between adjoining areas of color. Border distinctness is insensitive to blue-yellow differences from S-cone input (Tansley \& Boynton, 1976) and would otherwise introduce a confounding factor (as in Stalmeier \& de Weert, 1988). The gaps between the lozenges also reduce any luminance artifacts from chromatic aberration of the lens. 
Let $\mathrm{E}_{i}$ indicate the stimulus used to color the horizontal lozenges (as is shown in Figure 1), and let $\mathrm{E}_{j}$ and $\mathrm{E}_{k}$ indicate the stimuli used to color the two classes of oblique lozenge. Assume that $\mathrm{E}_{j}$ and $\mathrm{E}_{k}$ are the most similar pair in this triad (i.e., that the dissimilarity between them, $d_{j k}$, is less than the dissimilarities $d_{i j}$ and $d_{i k}$ ), whereas $\mathrm{E}_{i}$ is the "odd one out." The horizontal lozenges stand out in this case, forming an impression of narrow horizontal stripes, whereas the oblique lozenges fuse into broad horizontal stripes. In the two cases in which the dominant alignment is oblique, the subject's task is to rotate the pattern to a horizontal alignment (by pressing the arrow keys, which permutes the color mapping).

One hundred twenty of these triads were generated randomly and presented in sequence (with no time limit on the responses). This was done by forming five triads from the 17 stimuli used in this study, with two left over until the next iteration, performing this process 24 times. Quilt, a program for presenting triads and capturing responses, was written for IBM PCs (in Borland Pascal) and later run on a Mac Powerbook in its PC-emulation mode.

The stimuli were presented at a comfortable brightness level, somewhere in the middle range of monitor capability and clearly visible against the reflected ambient light, but were not otherwise quantified. Nor was ambient lighting itself controlled.

Any differences in brightness stand out in the triads and dominate the dissimilarities. Therefore, it is essential to adjust the stimuli to equal luminance (especially for protanopic or protanomalous subjects, who are relatively insensitive to long-wavelength light). ${ }^{2}$ In the initial stage of a session, heterochromatic flicker photometry was used to equate the luminance of each stimulus against a neutral gray. Isoluminance can potentially be disrupted by the drifting of the electron gun intensities over time, but for a 20-min session recalibration was not thought necessary. This does not address the problem that a pair of colors of equal luminance in the fovea may not be so in perifoveal vision, because of variations in the spectral sensitivity function across the retina. Similar nonhomogeneity of spectral emission may also be present across the screen.

\section{Stimuli}

We opted to use stimuli corresponding to samples of pigments that are standardized and widely accessible because they are used in panel tests for color vision deficiency and are therefore available for collecting conventional triad data (should confirmatory tests be necessary). In this case, the 17 Munsell papers constituting the H16 test were used. These have a value (lightness) of 5, traverse several levels of chroma (saturation), and trace an irregular arc through the color plane from yellow-red through red, purple, blue, green, and back to yellow-green, though any examples of yellow are lacking. Chromaticity coordinates were taken from Paulson (1973) and rendered on the computer screen. The coordinates of the $i$ th stimulus, $\mathrm{E}_{i}$, were converted into $\mathrm{R}, \mathrm{G}$, and B intensities $\left(r_{i}, g_{i}\right.$, and $b_{i}$, respectively) by solving the simultaneous equation:

$$
\mathrm{E}_{i}=r_{i} \mathbf{X}_{r}+g_{i} \mathbf{X}_{g}+b_{i} \mathbf{X}_{b},
$$

where $\mathbf{X}_{r}, \mathbf{X}_{g}$, and $\mathbf{X}_{b}$ are chromaticity coordinates for the individual phosphors (measured for a Sony Trinitron CRT using a Colortron digital photometer).

These intensities were converted to voltage settings for the monitor's three electron guns and rounded off to 6-bit precision (a limitation imposed by the programming environment). Rather than ascertain the nonlinear intensity/voltage function of each gun for a given monitor, the functions were assumed to be power functions with an exponent of $\gamma=2.4$. To ensure isoluminance across the stimuli, each triplet of values $r_{i}, g_{i}$, and $b_{i}$ was increased or decreased by a factor of $l_{i}$ while flicker photometry was used against a standard gray to equalize luminance of the $\mathrm{E}_{i}$.

It is worth noting that stimuli on a computer monitor are mixtures of the sharply peaked emission spectra of its phosphors. One cannot guarantee that a color-deficient observer's anomalous spectral sen- sitivity will affect perception of them in the same way that it would affect the broad-band reflected-light spectra of Munsell papers.

A monitor's R, G, and B phosphors mark the corners of a triangle in the color plane, defining the color gamut accessible to that monitor. As is described below, data collection involved a range of computers. Differences between manufacturers in the composition and characteristics of these phosphors imply that a stimulus located within the gamut by a particular combination of phosphor excitation will not be reproduced identically on monitors that deviate from these phosphor specifications.

\section{Subjects}

Fifteen subjects were recruited informally and informed in advance of the nature of the procedure and that they could withdraw their consent at any stage. In one condition, the test was performed by 5 of the subjects 10 times on a Mac Powerbook. Three of these subjects described themselves as RGD (differential diagnoses as protan or deutan were performed with the D15 panel test). One norma trichromat (author J.K.) performed the test five times, and the other performed it twice.

In the second condition, the test was performed by 10 of the subjects 15 times on a variety of PC monitors. Two of these subjects described themselves as RGD and one as borderline RGD (their selfreports of earlier diagnoses as protan or deutan were accepted). One NT, 1 RGD, and the borderline RGD used their own computers and returned the data via e-mail after contact was made through a Usenet discussion group. Author D.B. performed the test six times on four different monitors, including once with a finer tessellation (the lozenges measuring eight pixels per edge, to test the importance of scale) and once using the Stalmeier-de Weert Star of David gestalt against a black background (with elements separated by a 3-pixel black line).

In a further PC application of Quilt, 12 male and 8 female students were recruited by word of mouth as part of a larger study conducted to examine the possibility that cigarette smoking affects color vision. All were in the age range of 18-23 years, with the exception of 129 year-old, and all were smokers. Eighteen students performed the test twice, whereas 2 performed it three times. The same PC monitor (a Proview $768 \mathrm{M}$ ) was used throughout these 42 tests, with the same screen settings and the same level of ambient lighting each time.

The subjects performed the task once immediately after smoking a cigarette and again after several hours of not smoking (to check for short-term effects). The subjects were not paid and were unaware of the purpose of the test.

\section{Analysis}

Programs for MDS are legion, but they all share the goal of arranging points (representing the stimuli) so that the distances between them match as accurately as possible the dissimilarities among stimuli. This is an iterative process of rearranging points to progressively improve the accuracy of the match. MTRIAD, the program used here, is similar to Takane's (1978) MAXSCAL; it defines accuracy in terms of likelihood. The likelihood that the arrangement will give rise to a given triadic response is enhanced if the three stimuli form an elongated triangle in the solution, with the odd stimulus at its acute corner (Bimler \& Kirkland, 2001; Bimler et al., 2000). In other words, if $\mathrm{E}_{j}$ and $\mathrm{E}_{k}$ are most similar whereas $\mathrm{E}_{i}$ is the odd one out, MTRIAD tries to satisfy the comparisons $d_{j k}<d_{i j}$ and $d_{j k}<d_{i k}$.

One hundred twenty triads per subject are too few to derive individual MDS solutions, so we pooled the subjects' data and solved for a consensus color space: 17 points, located by a set of coordinates, which we write as the matrix $\mathbf{X}_{0}$. However, the color perception of individuals may deviate from that consensus. We accommodate any deviations by assuming $\mathbf{X}_{m}$, the perceptual space of Subject $m$, to be a distorted version of $\mathbf{X}_{0}$. A simple form of distortion allows $\mathbf{X}_{0}$ to be compressed along one axis (or, equivalently, elongated along the other axis or axes). The axes are the same for all the subjects. Their 


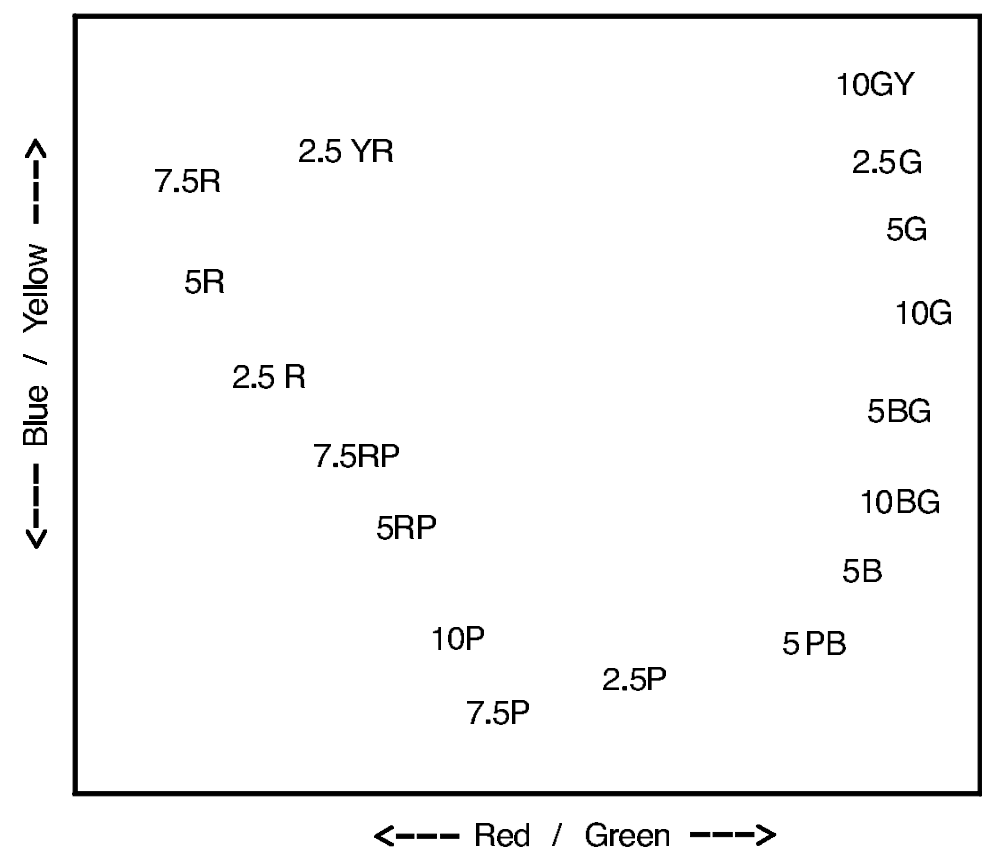

Figure 2. D1/D2 plane of MDS solution for 17 color stimuli.

direction - that is, the orientation of $\mathbf{X}_{0}$-is not assumed in advance, emerging instead from the analysis. This is the weighted Euclidean model, which basically assumes that individuals vary by paying relatively more or less attention to interstimulus differences along particular axes. It corresponds to the INDSCAL model of individual difference, used when metric MDS is applied to interval-level data (Wish \& Carroll, 1974).

For a 2-D solution, let $w_{m 1}$ and $w_{m 2}$ represent the compression parameters (i.e., dimensional saliences) for Subject $m$, with the constraint that $w_{m 1}^{2}+w_{m 2}^{2}=2$ (to maintain the overall scale of $\mathbf{X}_{m}$ ). Consider a hypothetical observer who perceives no difference between stimuli on one side of $\mathbf{X}_{0}$ and their counterparts on the other (e.g., the points $10 \mathrm{GY}$ and 2.5YR in Figure 2). In any triad involving such a pair of stimuli, the third stimulus would always be the odd one out. A limiting case of this kind would be represented in this geometric framework by compressing $\mathbf{X}_{m}$ so that the points representing the indistinguishable stimuli coincide (i.e., $w_{m 1}=0$ ).

In general, the optimal $w_{m 1}$ and $w_{m 2}$ values are those that maximize the tendency for the odd stimuli in each of Subject $m$ 's triad decisions to form the apex of the corresponding triangle in $\mathbf{X}_{m}$ (naturally, the triangles are differently elongated in comparison with their counterparts in $\mathbf{X}_{0}$, perhaps in different directions). In three dimensions, there are three parameters, such that $w_{m 1}^{2}+w_{m 2}^{2}+w_{m 3}^{2}=3$.

A more general form of distortion allows $\mathbf{X}_{0}$ to undergo a rotation to a subject-specific set of axes prior to compression. The angle of rotation $\theta_{m}$ is another subject parameter in this idiosyncratic-weights model (Bimler et al., 2000; Paramei et al., 2001). It corresponds to the IDIOSCAL model in the case of metric MDS (Wish \& Carroll, 1974). Only rotations in the D1/D2 plane were considered. Ambiguities were eliminated by assuming that $w_{m 1} \leq w_{m 2}$ and that $-90^{\circ}<\theta_{m} \leq 90^{\circ}$. Compression along the first axis (D1) corresponds to $\theta_{m}=0$, whereas compression along the second axis (D2) is equivalent to a rotation of $\theta_{m}=90^{\circ}$ prior to compression. Note that the relative values of $\theta_{m}$ are meaningful, but their absolute values are not: A constant angle $\phi$ can be added to all $\theta_{m}$ without affecting $\mathbf{X}_{m}$, as long as $\mathbf{X}_{0}$ is rotated through $-\phi$.

It is convenient to introduce a measure of color-space compression $\left[r_{m}=\left(w_{m 2}^{2}-w_{m 1}^{2}\right) /\left(w_{m 2}^{2}+w_{m 1}^{2}\right)\right]$. The uncompressed case (where $w_{m 1}=w_{m 2}$ ) is $r_{m}=0$. Conversely, the hypothetical case of dichromacy, in which the color plane collapses to a line in some direction (because $w_{m 1}=0$ ), is $r_{m}=1$.

\section{RESULTS}

Stimulus isoluminance was not exact for the seven trials of the NT subjects running Quilt on the same Mac Powerbook. This was a result of haste on the authors' part: Convinced by the IBM PC trials that the stimuli were isoluminant for NTs, they omitted the initial stage of flicker photometry in this condition. In fact, because of obscure hardware reasons, two stimuli were slightly brighter, and the 2-D MDS solution derived from Powerbook data sets deviated from the solution derived from the IBM PC data (with a displacement of those two points). The discrepancy was handled by retaining a $3-D \mathbf{X}_{0}$ to allow a displacement along the third axis (D3) of the points representing those two stimuli. According to individual differences analysis, D3 contributes only to the seven Powerbook trials of NTs ( $w_{m 3}$ is small in other cases) and can be ignored in analyses that omit those data. Our confidence in the analysis is bolstered by its detection of this error.

The first two dimensions of $\mathbf{X}_{0}$ (see Figure 2) accurately recover the arrangement of the stimuli in the color plane, as is shown in Paulson's (1973) Figure 5.

We turn now to individual differences. The 25 data sets from the first and second groups of subjects ( 10 data sets for which one Powerbook was used and 15 for which miscellaneous IBM PC monitors were used) were analyzed with the weighted Euclidean model. For a 3-D $\mathbf{X}_{0}$, there are three parameters of dimensional salience per subject, but these are interdependent because their squares must add 


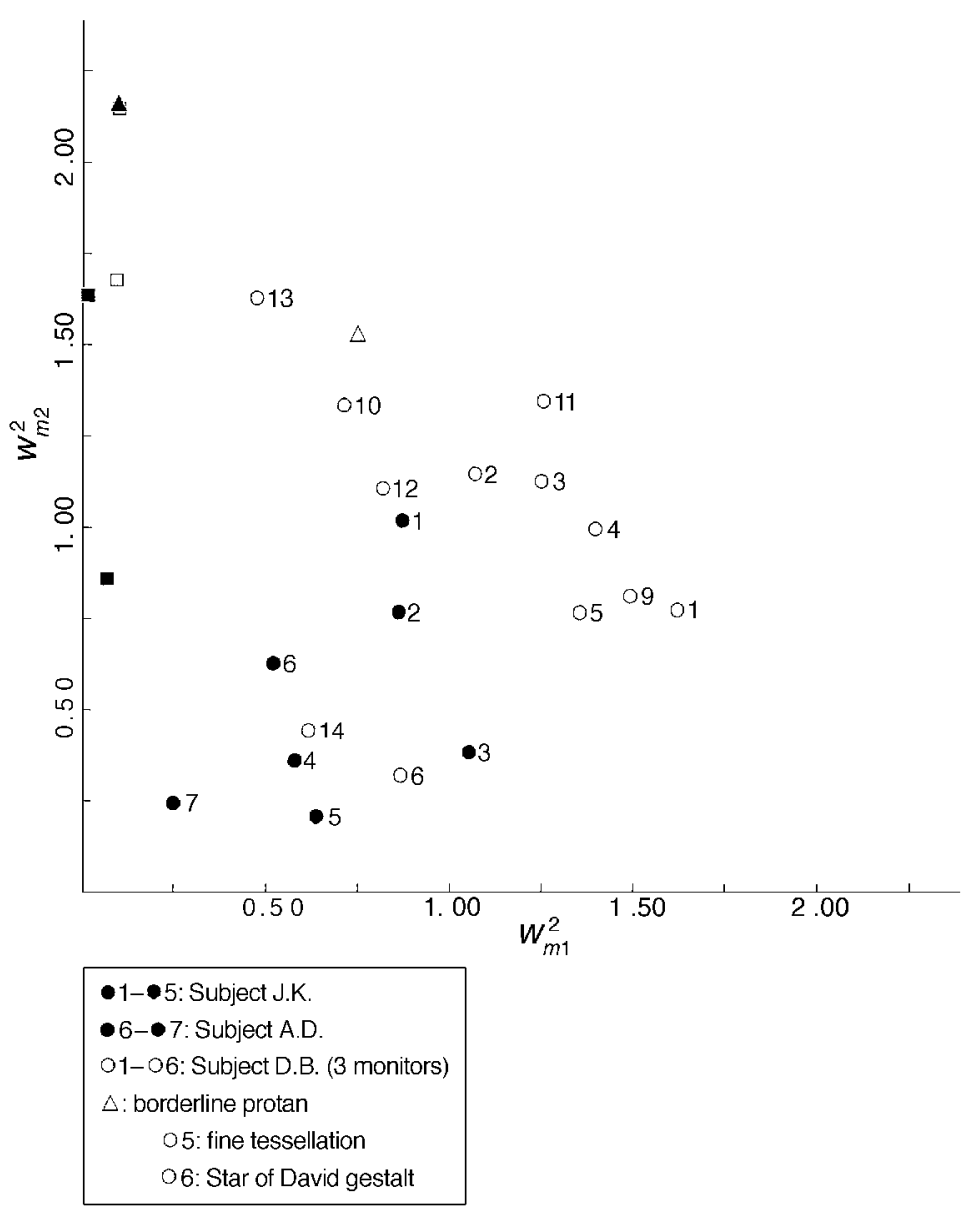

Figure 3. Weighted Euclidean subject parameters for 25 data sets. The horizontal and vertical axes mark $w_{m 1}^{2}$ and $w_{m 2}^{2}$ (i.e., salience of red-green and blue-yellow directions in color space), respectively. Clustering near the ordinate of symbols for the RGD subjects reflects the red-green compression of their personal color spaces. Circles, triangles, and squares denote the data of NTs, protans, and deutans, respectively. Solid symbols represent the data of subjects using the same Mac Powerbook, and open symbols represent the data of subjects using a variety of PC monitors.

up to 3. Subject parameters are plotted in Figure 3, with $w_{m 1}^{2}$ and $w_{m 2}^{2}$ as the abscissa and the ordinate, respectively: As these two values decrease, for points close to the origin, $w_{m 3}$ increases correspondingly.

Red-green deficiency is associated with a low $w_{m 1}: w_{m 2}$ ratio. Clearly, the RGD subjects fit the geometrical paradigm of color-space compression; their patterns of oddone-out responses imply that their personal spaces are compressed along the red-green direction, so that color differences in this direction contribute little to the dissimilarities they perceive. This is recognized by the special status of the red-green direction as the D1 axis of $\mathbf{X}_{0}$. The borderline protanope is represented by the triangle located between the NTs and the RGDs. The subject represented by the symbol $\odot 13$ in the same area was not aware of any deficit.
As was noted above, high $w_{m 3}$ values characterize the data sets collected from NT subjects using a Powerbook (shown in Figure 3 as solid circles near the origin), in contrast with those collected from the PC users (shown as hollow circles), for whom D3 contributed little to interitem dissimilarities and to odd-one-out decisions. Within the former group, the data sets $\bullet 1-\bullet$ from Subject J.K. show a good degree of consistency and appear to differ from sets $\bullet 6$ and $\bullet 7$ from Subject A.D. (the latter is no longer available to provide more data to verify this). Within the latter group, there is equally good consistency among the data sets $\bigcirc 1-\bigcirc$, collected from Subject D.B. on a range of monitors. The parameters obtained with a fine tessellation $(\bigcirc 5)$ are part of the cluster, suggesting that tessellation coarseness is not crucial. Variations in the nature of the gestalt do not seem to be crucial either: Data obtained 


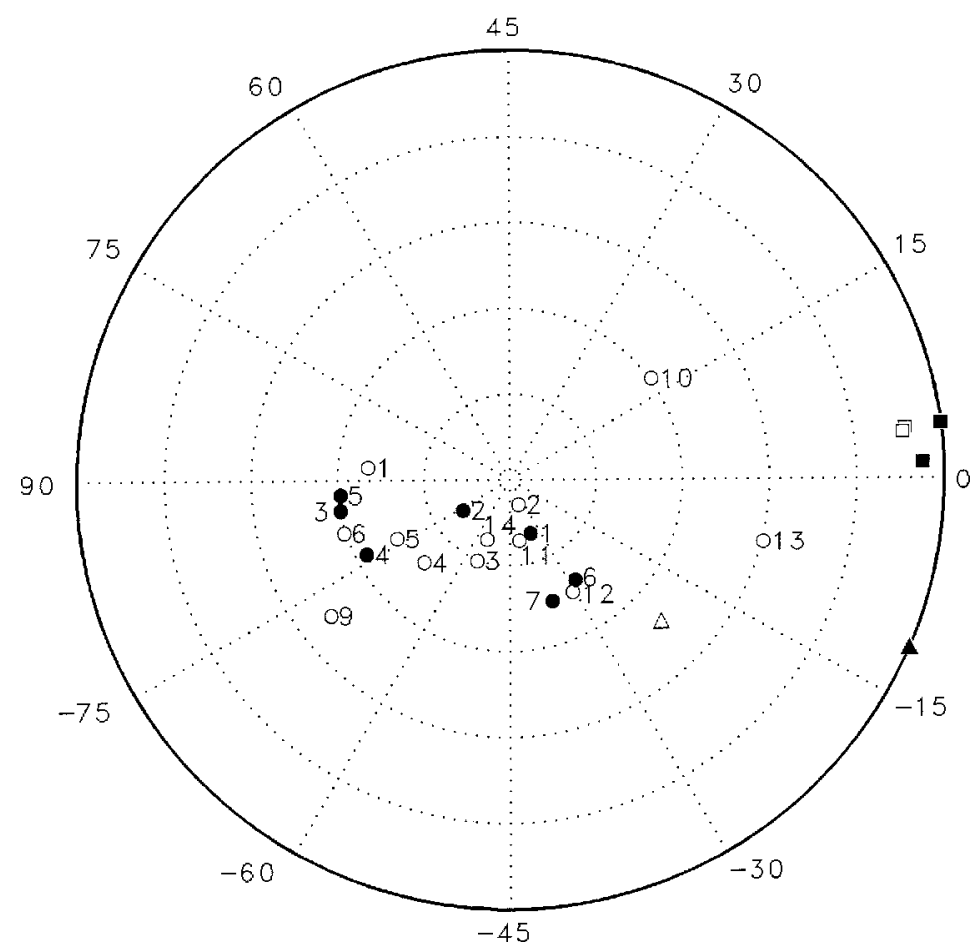

Figure 4. Idiosyncratic-weights subject parameters, plotted in polar coordinates $2 \theta_{m}$ and $r_{m}$. Data sets and symbols are as in Figure 3 .

with the Star of David gestalt $(\bigcirc 6)$ lead to parameters that are distinguished from others for Subject D.B., primarily by a high $w_{m 3}$ value.

In Figure 4, the subject parameters from the idiosyncratic weights model are plotted in polar coordinates, with $2 \theta_{m}$ and $r_{m}$ as the angular and radial coordinates, respectively. The alignment of $\mathbf{X}_{0}$ was retained from the weighted Euclidean solution. It becomes evident that protans and deutans are characterized by slightly different axes of compression and that the red-green dimension D1 is a compromise between these. The $\theta_{m}$ parameter clearly distinguishes the two groups.

Finally, analysis of the 42 sets of data from the third group (consisting of 20 NTs) yielded a 2 -D $\mathbf{X}_{0}$ very similar to that presented in Figure 2. Application of the idiosyncratic weights model revealed that $r_{m}<.4$ for all but two data sets. To quantify the stability of subject parameters between test and retest, we defined an index of discordance, $\mathrm{D}_{m n}$, between the parameters for the $m$ th and $n$th data sets. $\mathrm{D}_{m n}$ is simply the straight-line distance between points in the polar-coordinate plot:

$$
\begin{aligned}
\mathrm{D}_{m n}^{2}= & {\left[r_{m} \cos 2 \theta_{m}-r_{n} \cos 2 \theta_{n}\right]^{2} } \\
& +\left[r_{m} \sin 2 \theta_{m}-r_{n} \sin 2 \theta_{n}\right]^{2} .
\end{aligned}
$$

Across the cases in which $m$ and $n$ refer to the same subject, the mean $\mathrm{D}_{m n}$ is 0.26 , in comparison with a mean value of 0.33 across the cases in which $m$ and $n$ refer to different subjects. $\mathrm{D}_{m n}$ is significantly smaller in the for- mer case $(p<.05)$, although the difference is not enormous. We suspect that the unmotivated state of this group of subjects contributed to the relatively high test/retest discordance, which is only an upper limit.

Erb et al. (1999) found that cigarette smoking can affect color vision. If any short-term effect exists, it would have inflated the test/retest discordances. However, no systematic group differences could be discerned between performance before and performance after smoking.

No group difference was found between the parameters of males and those of females.

\section{DISCUSSION}

\section{An Initial Caveat}

Because of variations among monitors (not to mention inexact compensation for nonlinearity of intensity as a function of voltage), the stimuli in each session are only approximate reproductions of the stimuli in other sessions and of the hues used in the H16. ${ }^{3}$ The gamut of stimuli that can be presented on a given monitor lies within a triangle in the color plane, with the locations of the three phosphors as its corners. Translation of the gamut would be a concern in experiments conducted in the study of the properties of individual stimuli (such as the names given to them), but, fortunately, it is not an issue here, because translation does not affect the pattern of relationships among stimuli. This is assuming that the shape of the 
gamut is not greatly perturbed by displacement of its corners. Thus, the stimuli were specified relative to the gamut, but this was done as precisely as possible so that data sets could be combined with confidence.

The compound display used here yielded results (for author D.B.) similar to those of the Star of David stimulus display pioneered by Stalmeier and de Weert $(1988,1994)$, but, subjectively, the decisions were more clear-cut, presumably facilitated by the repetition of the basic elements. The Star of David display was modified by separating its elements with a black line to prevent border distinctness from contributing to the dissimilarities. Another problem-less easily remedied - is that for some combinations of colors, a transparency gestalt competes with the fusion gestalts. The Star of David is perceived as one translucent triangle overlapping another that is partly visible through it. This problem does not arise in the tessellation of Figure 1.

One hundred twenty triads collected in this way provide a sufficiently large signal-to-noise ratio to distinguish RGDs from NTs when analyzed with the weighted Euclidean model. Though this confirms previous analyses of visiontest responses (Bimler et al., 2000), it is hardly surprising, in that a model of geometrical compression underlies the tests themselves (Farnsworth, 1943). Standard tests for differential diagnosis among RGDs relies on the fact that they are a nonhomogeneous group, with protans and deutans exhibiting color-space compression along different angles (the red-green axis of Figure 2 being a compromise value). In Figure 2 , the $\theta_{m}$ parameter of the idiosyncratic weights model served to separate the two subgroups. This was the case in previous applications (Paramei et al., 2001) and in a reanalysis of Helm's (1964) data.

Note that protan and deutan subgroups are not internally homogeneous; within each subgroup there is a distribution of $\theta_{m}$ values around a modal value. For lack of retests, the present study cannot specify how much of this is noise, but Bimler et al. (2000) obtained multiple values for RGDs who had taken several tests and found them to be significantly correlated (for protans and for deutans separately).

The framework of individually compressed color spaces also provides a parsimonious description of variations among NTs. They are spread along a spectrum of dimensional saliences comparable to the range found by Wish and Carroll (1974).

Smith and Pokorny (1995) considered how much the salience and direction of cone-excitation axes could vary between subjects, extrapolating sources of variation identified by factor analysis of color-matching data (Webster \& MacLeod, 1988). Such variations have also been studied empirically (Webster, Miyahara, Malkoc, \& Raker, 2000). Presumably, the axial rotation and obliquity in these studies apply to RGD as well as to NT observers.

The axes emerging here from the weighted Euclidean model are chosen to account for the maximum amount of variation between observers. They are therefore dominated by the difference between NTs and RGDs, although they are sufficiently relevant to variations among NTs to separate them reliably. Moreover, the same axes emerge from weighted Euclidean treatment of the NTs alone. They are not necessarily identical to the framework of coneexcitation axes in which Smith and Pokorny (1995) and Webster et al. (2000) expressed their results.

It has been pointed out to the authors that the tessellation of Figure 1 lends itself to an alternative gestalt. For some color combinations, the visual system may find it parsimonious to interpret Figure 1 as a repeated 3-D pattern of cubes more or less uniform in hue (each alignment of lozenges being a surface plane of squares seen in perspective), with one of the surface planes altered by light of another hue shining from one direction-from above if $\mathrm{E}_{i}$ is the odd one out. This percept increases the subjective brightness of those lozenges, thereby disrupting the isoluminance of the stimuli. Fortunately, this potentially confounding factor is not in conflict with the primary effect of fusion into stripes. At most, it enhances the dissimilarity of the stimulus that is already most dissimilar.

Roberson and Davidoff (2000) showed that color dissimilarities are not judged on purely perceptual bases but are biased by semantic knowledge, unless subjects simultaneously perform a verbal task to interfere with verbal coding of stimuli. Triadic comparisons with gestalt displays are another way of bypassing semantic processing, particularly when the stimuli are nonprototypal colors, as is the case here.

\section{REFERENCES}

Bimler, D., \& KirKLAND, J. (2001). Categorical perception of facial expressions of emotion: Evidence from multidimensional scaling. Cognition \& Emotion, 15, 633-658.

Bimler, D., KiRKLAND, J., \& JACOBS, R. (2000). Colour-vision tests considered as a special case of multidimensional scaling. Color $R e$ search \& Application, 25, 160-169.

COWAN, W. B. (1983). An inexpensive scheme for calibration of a colour monitor in terms of CIE standard coordinates. Computer Graphics, 17, 315-321.

Erb, C., Nicaeus, T., Adler, M., Isensee, J., Zrenner, E., \& Thiel, H.-J. (1999). Colour vision disturbances in chronic smokers. Graefe's Archive of Clinical \& Experimental Ophthalmology, 237, 377-380.

FARnsworTH, D. (1943). The Farnsworth-Munsell 100-hue and dichotomous tests for color vision. Journal of the Optical Society of America, 33, 568-578.

HeLM, C. R. (1964). Multidimensional ratio scaling analysis of perceived color relations. Journal of the Optical Society of America, 54, 256-262.

Indow, T. (1988). Multidimensional studies of Munsell color solid. Psychological Review, 95, 456-470.

INDOW, T., \& KANAZAWA, K. (1960). Multidimensional mapping of Munsell colors varying in hue, chroma and value. Journal of Experimental Psychology, 59, 330-336.

Papathomas, T. V., Kovács, I., Gorea, A., \& Julesz, B. (1995). A unified approach to the perception of motion, stereo, and static-flow patterns. Behavior Research Methods, Instruments, \& Computers, 27, 419-432.

Paramei, G. V., Bimler, D. L., \& Cavonius, C. R. (2001). Color-vision variations represented in an individual-difference vector chart. Color Research \& Application, 26, S230-S234.

PAulson, H. M. (1973). Comparison of color vision tests used by the armed forces. In D. B. Judd (Ed.), Color vision (pp. 34-64). Washington, DC: National Academy Press.

Richardson, M. W. (1938). Multidimensional psychophysics. Psychological Bulletin, 35, 659-660. 
RobERSON, D., \& DAVIDOFF, J. (2000). The categorical perception of colors and facial expressions: The effect of verbal interference. Memory \& Cognition, 28, 977-986.

Smith, V. C., \& Pokorny, J. (1995). Chromatic-discrimination axes, CRT phosphor spectra, and individual variation in color vision. Journal of the Optical Society of America A, 12, 27-35.

StAlmeier, P. F. M., \& DE WeERT, C. M. M. (1988). Large colour differences measured by spontaneous Gestalt formation. Color Research \& Application, 13, 209-218.

Stalmeier, P. F. M., \& DE WeERT, C. M. M. (1994). On the conditions affecting the contribution of color to brightness perception. Color Research \& Application, 19, 192-201.

TAKANE, Y. (1978). A maximum likelihood method for nonmetric multidimensional scaling: I. The case in which all empirical pairwise orderings are independent-Theory. Japanese Psychological Research, 20, 7-17.

TANSLEy, B. W., \& Boynton, R. M. (1976). A line, not a space, represents visual distinctness of borders formed by different colors. Science, 191, 954-957.

TAYLOR, J. M., \& Billme YER, F. W., JR. (1988). Multidimensional scaling of selected samples from the Optical Society of America Uniform Color Scales. Color Research \& Application, 13, 85-98.

TORGERSON, W. S. (1958). Theory and methods of scaling. New York: Wiley.

WeBster, M. A., \& MACLEOD, D. I. A. (1988). Factors underlying individual differences in the color matches of normal observers. Journal of the Optical Society of America A, 5, 1722-1736.

Webster, M. A., Miyahara, E., Malkoc, G., \& Raker, V. E. (2000). Variations in normal color vision: I. Cone-opponent axes. Journal of the Optical Society of America A, 17, 1535-1544.
WISH, M., \& CARROLL,J. D. (1974). Applications of individual differences scaling to studies of human perception and judgment. In E. C. Carterette \& M. P. Friedman (Eds.), Handbook of perception (Vol. 2, pp. 449-491). New York: Academic Press.

WRIGHT, H. (1965). Precision of color differences derived from a multidimensional scaling experiment. Journal of the Optical Society of America, 55, 1650-1655.

Wuerger, S., Maloney, L. T., \& Krauskopf, J. (1995). Proximity judgments in color space: Tests of a Euclidean color geometry. Vision Research, 35, 827-835.

\section{NOTES}

1. This is similar to the forced-choice competition paradigm, in which a compound stimulus, interpretable in two mutually incompatible ways, is used to collect data that are in effect triadic. Applications include comparisons of the relative importance of color and luminance in several visual modalities (Papathomas, Kovács, Gorea, \& Julesz, 1995).

2. For lack of better terms, here we use luminance and isoluminant in the sense of subject luminance, adjusted to the individual's own spectral efficiency function. This is distinct from CIE luminance, defined in terms of a standard observer with a standard efficiency function.

3. Cowan (1983) called for monitors to be calibrated carefully because of the lack of standardization among them. More recently, Smith and Pokorny (1995) measured four monitors (all based on Trinitron CRTs from Sony, a variety widely used in laboratories) and found their phosphor chromaticity to be indistinguishable.

(Manuscript received May 28, 2002; revision accepted for publication March 28, 2003.) 